SLAC-PUB-8796

SU-ITP 01-10

hep-th/0103179

\title{
Quantum Hall Physics Equals Noncommutative Field Theory
}

\author{
Simeon Hellerman ${ }^{a}$ and Mark Van Raamsdonk ${ }^{b}$ \\ ${ }^{a, b}$ Stanford Linear Accelerator Center \\ Stanford, CA 94305 U.S.A. \\ simeon@itp. stanford.edu \\ ${ }^{b}$ Department of Physics \\ Stanford University \\ Stanford, CA 94305 U.S.A. \\ mav@itp.stanford. edu
}

\begin{abstract}
In this note, we study a matrix-regularized version of non-commutative $U(1)$ ChernSimons theory proposed recently by Polychronakos. We determine a complete minimal basis of exact wavefunctions for the theory at arbitrary level $k$ and rank $N$ and show that these are in one-to-one correspondence with Laughlin-type wavefunctions describing excitations of a quantum Hall droplet composed of $N$ electrons at filling fraction $1 / k$. The finite matrix Chern-Simons theory is shown to be precisely equivalent to the theory of composite fermions in the lowest Landau level, believed to provide an accurate description of the filling fraction $1 / k$ fractional quantum Hall state. In the large $N$ limit, this implies that level $k$ noncommutative $U(1)$ Chern-Simons theory is equivalent to the Laughlin theory of the filling fraction $1 / k$ quantum Hall fluid, as conjectured recently by Susskind.
\end{abstract}




\section{Introduction}

In its simplest form, noncommutative geometry is characterized by a pair of coordinates which do not commute in the same sense that canonically conjugate positions and momenta do not commute in quantum mechanics,

$$
[x, y]=i \theta
$$

where $\theta$ is a dimensionful parameter. Over the past few years, it has become increasingly apparent that noncommutative geometry plays an important role in string theory $[1,2]$. However, such a commutation relation also appears in a much simpler context, that of charged particles in a strong magnetic field.

For a single particle of unit charge moving in two dimensions under the influence of a constant magnetic field, the Lagrangian is given by

$$
L=\frac{m}{2}\left(\dot{x}^{2}+\dot{y}^{2}\right)+\frac{1}{2} B(\dot{x} y-\dot{y} x) .
$$

It is well known that the eigenstates of this system lie in Landau levels, degenerate sets of states at energies given by $E_{n}=\left(n+\frac{1}{2}\right) \frac{B}{m}$. In each level, the degeneracy is equal to one state for each unit of area defined by the inverse density of flux quanta. In the limit of very large magnetic field, particles are restricted to the lowest Landau level, and the physics may be described by ignoring the first term in (2). Canonically quantizing the resulting system, we are led to precisely the commutation relation (1) with $\theta$ replaced by $\frac{1}{B}$. Thus, the two dimensional coordinate space becomes the phase space for the system and gives a simple realization of a noncommutative geometry.

The physics of electrons in the lowest Landau level exhibits many fascinating properties. In particular, when the electron density lies at certain rational fractions of the density corresponding to a fully filled lowest Landau level, the electrons condense into special incompressible fluid-like states whose excitations exhibit such unusual phenomena as fractional charge and fractional statistics. These states also have a gap in their excitation spectrum which gives rise to the experimentally observed fractional quantum Hall effect (for a recent review, see [3] and references therein). For the filling fractions $1 / k$, the physics of these states is accurately described by certain wavefunctions proposed by Laughlin, and more general wavefunctions may be written down describing the various types of excitations about the Laughlin states.

Given that the first quantized system of a particle in a strong magnetic field naturally realizes a noncommutative space, it is interesting to speculate that the second-quantized field theory description of the Quantum Hall fluid for various filling fractions might involve a noncommutative field theory. In a recent paper by Susskind [4], this possibility was put forward in a precise conjecture, that the Laughlin state of electrons at filling fraction $1 / k$ is precisely described by the noncommutative version of $U(1)$ Chern-Simons theory at level $k$. Subsequently, Polychronakos [5] proposed that a particular matrix regularized 
version of this level $k$ noncommutative Chern-Simons theory could be used to describe a finite number of electrons in a "droplet" of the Quantum Hall fluid at filling fraction $1 / k$.

In this paper, we cement the connection between noncommutative Chern-Simons theory and the Laughlin theory of the Quantum Hall fluid. Specifically, write down the complete set of exact wavefunctions of the Polychronakos model and show that they are in one-to-one correspondence with wavefunctions describing excitations of a filling fraction 1/ $k$ Quantum Hall droplet in the Laughlin theory (the Hilbert spaces are isomorphic and the energy levels are the same). By taking the limit of a large number of electrons, this should imply that the filling fraction 1/ $k$ Quantum Hall fluid of infinite extent is well described by the noncommutative $U(1)$ Chern-Simons theory at level $k$, as conjectured by Susskind.

The plan of the paper is as follows. In section 2, we review some basic properties of charged fermions in the lowest Landau level. We review Laughlin's wavefunctions for the filling fraction $\nu=1 / k$ states as well as the more general wavefunctions describing excitations about these states. In section 3, we describe the noncommutative ChernSimons theory and its matrix description, and review some of the evidence put forward by Susskind that this theory describes the fractional quantum Hall fluid. In section 4, we present the finite dimensional matrix Chern-Simons theory proposed by Polychronakos and review its quantization. Section 5 contains the main results of this paper: we write down explicitly the wavefunctions for a complete minimal basis of energy eigenstates for the theory and show that they are in one-to-one correspondence with wavefunctions describing excitations of a quantum Hall droplet described by the Laughlin theory at $1 / k$. In section 6 , we offer some concluding remarks.

\section{Charged particles in the lowest Landau level}

In this section, we review some aspects of the physics of charged fermions in a strong magnetic field. We recall Laughlin's wavefunctions describing particles in the lowest Landau level at filling fractions $1 / k$ as well as the more general wavefunctions describing excitations about these states.

We begin by considering a single particle of mass $m$ and unit charge in a magnetic field $B$. It is convenient to break the degeneracy arising from translation invariance, so we also include a harmonic oscillator potential (which we may take to be arbitrarily weak). The complete Lagrangian is then given (in the radial gauge $A_{i}=\frac{1}{2} B \epsilon^{i j} x^{j}$ ) by

$$
L=\frac{m}{2} \dot{x}_{i}^{2}+\frac{B}{2} \epsilon^{i j} \dot{x}_{i} x_{j}-\frac{1}{2} \kappa x_{i}^{2} .
$$

The energy eigenstates for this system may be determined exactly. In the limit $m \rightarrow$ 0 (equivalent to strong magnetic field), the wavefunctions whose energies remain finite 
relative to the ground state energy may be labeled by an integer $n \geq 0$ and are given by

$$
\langle\vec{x} \mid n\rangle=\sqrt{\frac{B^{n+1}}{2^{n+1} \pi n !}} z^{n} e^{-\frac{B}{4}|z|^{2}}
$$

where $z=x+i y$. These have energies $E_{n}=\frac{\kappa}{B}\left(n+\frac{1}{2}\right)$.

Another way to arrive at these states is by taking $m=0$ at the start. Then the canonical commutation relations give

$$
[x, y]=\frac{i}{B}
$$

and the Hamiltonian is that of a Harmonic oscillator,

$$
H=\frac{\kappa}{B}\left(a^{\dagger} a+\frac{1}{2}\right)
$$

where we have defined $a=\sqrt{\frac{B}{2}}(x+i y)$ so that $a$ and $a^{\dagger}$ have the usual commutation relations of creation and annihilation operators. The eigenstates, again labeled by $n$, are of course

$$
|n\rangle=\frac{1}{\sqrt{n !}}\left(a^{\dagger}\right)^{n}|0\rangle
$$

with energies as above.

It will be helpful to understand the precise relationship between these wavefunctions (6), which depend only on a single variable, and those in (4) which depend on two coordinates. In the reduced system, we may define a coherent state basis $|z\rangle$ by $a|z\rangle=z|z\rangle$. In this basis, we have $\langle z \mid n\rangle=\frac{1}{\sqrt{n !}} z^{n}$, so we see that the analytic part of the lowest Landau level wavefunctions (4) should be identified with the coherent state wavefunction $\langle z \mid \psi\rangle$ in the system with reduced phase space.

In the second picture, it is valuable to note that the hamiltonian $H$ is proportional to the radius squared operator, $H=\frac{1}{2} \kappa R^{2}$ and also generates rotations (because of the commutation relation (5)). Thus, the state labeled by $n$ has energy, angular momentum and $R^{2}$ all proportional to $n$.

Consider now the system of $N$ fermions, each described by the Lagrangian (3) in the limit of large magnetic field (we assume for now that their interactions may be ignored). The wavefunctions must be completely antisymmetric and it is easy to see that an orthogonal basis of energy eigenstates is provided by the set of wavefunctions

$$
\left|\left\{n_{i}\right\}\right\rangle=\epsilon^{i_{1} \cdots i_{N}} a_{i_{1}}^{\dagger} n_{1} \cdots a_{i_{N}}^{\dagger} n_{N}|0\rangle
$$

where $n_{1}<n_{2}<\cdots<n_{N}$. These correspond to states with one particle in each of the energy levels labeled by $n_{i}$, so the total energy is $E=\frac{\kappa}{B}\left(\frac{N}{2}+\sum n_{i}\right)$. In the original two dimensional language, these may be written as

$$
\left\langle\vec{x} \mid\left\{n_{i}\right\}\right\rangle=\epsilon^{i_{1} \cdots i_{N}} z_{i_{1}}^{n_{1}} \cdots z_{i_{N}}^{n_{N}} e^{-\frac{B}{4} \sum\left|z_{i}\right|^{2}} .
$$


The ground state corresponds to choosing $n_{i}=i-1$, so that the $R^{2}$ expectation value for the outermost particle is $R^{2}=\frac{2}{B}\left(N-\frac{1}{2}\right)$. The disc bounded by this radius contains $\sim N$ quanta of flux (the density of flux quanta is $B / 2 \pi$ ), so we see that the ground state corresponds to a circular "quantum hall droplet" with filling fraction $\nu=1$. Since the number of states per unit area in the lowest Landau level is equal to the number of flux lines per unit area, this ground state droplet is maximally dense and therefore incompressible. This may be seen directly by noting that the size of the droplet is independent of the strength of the harmonic oscillator potential. As a result, the external potential we have introduced should not significantly affect the physics of the droplet.

Using Fact 1 from the appendix, we may rewrite the $\nu=1$ ground state wavefunction $\left|\psi_{1}\right\rangle \equiv\left|n_{i}=i-1\right\rangle$ in a more standard form,

$$
\left\langle\vec{x} \mid \psi_{1}\right\rangle=\left(\prod_{i<j}^{N}\left(z_{i}-z_{j}\right)\right) e^{-\frac{B}{4} \sum\left|z_{i}\right|^{2}} .
$$

As required by antisymmetry, the wavefunction vanishes as any two particles become coincident. This also ensures that the wavefunction will continue to provide a good description in the presence of repulsive interparticle interactions as long as they are sufficiently weak/short-ranged.

Systems of electrons in a strong magnetic field also condense into special incompressible states at certain rational filling fractions, giving rise to the fractional quantum Hall effect. Generalizing the $\nu=1$ ground state wavefunction (9), Laughlin [6] proposed that for $\nu=$ $1 / k$, these fractional Quantum hall states could be accurately described by wavefunctions

$$
\begin{aligned}
\left\langle\vec{x} \mid \psi_{1 / k}\right\rangle & =\left(\prod_{i<j}^{N}\left(z_{i}-z_{j}\right)^{k}\right) e^{-\frac{B}{4} \sum\left|z_{i}\right|^{2}} \\
& =\left(\epsilon^{i_{1} \cdots i_{N}} z_{i_{1}}^{0} \cdots z_{i_{N}}^{N-1}\right)^{k} e^{-\frac{B}{4} \sum\left|z_{i}\right|^{2}} .
\end{aligned}
$$

It is easy to see that the highest power of a given $z_{i}$ appearing (proportional to the radius squared of the droplet) is $k(N-1)$, so this state has an electron density of $1 / k$ times the $\nu=1$ ground state, as required. The presence of an additional factor $\left(z_{i}-z_{j}\right)^{k-1}$ for each pair of particles relative to the $\nu=1$ state (and therefore an additional phase of $(-1)^{k-1}$ when the particles are interchanged) may be understood to arise from interparticle interactions which result in the binding of $k-1$ flux lines to each electron, forming a "composite fermion" $[7,8]$. This helps to minimize the repulsive Coulomb potential, as is evident from the rapid vanishing of the wavefunction as any two particles approach each other. The resulting composite fermions are weakly interacting and feel a reduced net magnetic field corresponding to the unattached flux lines. The density of composite fermions is precisely equal to the density of these unattached flux lines, so the $\nu=1 / k$ state may be interpreted as the $\nu=1$ state of the composite fermions. It is important 
to note that $k$ must be an odd integer in order to preserve the fermionic statistics of the particles (reflected in the antisymmetry of the wavefunction).

Based on this composite fermion picture, the general set of wavefunctions describing excitations of the $\nu=1 / k$ state should be obtained from the $\nu=1$ wavefunctions (8) simply by multiplying by the factor $\prod\left(z_{i}-z_{j}\right)^{k-1}$ corresponding to the flux attachment. Equivalently, the wavefunctions describing excitations of the $1 / k$ state may be taken to be the subset of energy eigenstates states spanned by the basis (8) which contain a zero of order at least $k$ when any pair of particles become coincident.

Using the harmonic oscillator representation (which will be most convenient for our later comparison), a minimal basis of wavefunctions describing excitations of the $\nu=1 / k$ state in the Laughlin theory is therefore given by

$$
\left|\left\{n_{i}\right\}, k\right\rangle=\epsilon^{i_{1} \cdots i_{N}} a_{i_{1}}^{\dagger} n_{1} \cdots a_{i_{N}}^{\dagger} n_{N}\left(\epsilon^{i_{1} \cdots i_{N}} a_{i_{1}}^{\dagger 0} \cdots a_{i_{N}}^{\dagger N-1}\right)^{k-1}|0\rangle
$$

where $n_{i}$ are integers such that $0 \geq n_{1}<\cdots<n_{N}$. The energies of these states is given by $E\left(\left\{n_{i}\right\}, k\right)=\frac{\kappa}{B}\left(\frac{N}{2}(1+(k-1)(N-1))+\sum n_{i}\right)$

Using Fact 2 from the appendix, it is not difficult to see that another minimal basis of wavefunctions is given by

$$
\left|\left\{c_{i}\right\}, k\right\rangle=\left(\sum a_{i}^{\dagger N}\right)^{c_{N}} \cdots\left(\sum a_{i}^{\dagger}\right)^{c_{1}}\left(\epsilon^{i_{1} \cdots i_{N}} a_{i_{1}}^{\dagger 0} \cdots a_{i_{N}}^{\dagger}{ }^{N-1}\right)^{k}|0\rangle
$$

where $c_{i}$ are arbitrary non-negative integers and the energy of these states is $E=\frac{\kappa}{B}\left(\frac{N}{2}(1+\right.$ $\left.k(N-1))+\sum m c_{m}\right)$.

In section 5, we will construct two bases of the wavefunctions for the finite $N$ matrix noncommutative Chern-Simons theory and see that they have a structure almost identical to the two bases constructed here. In preparation for this, we provide a brief review in the next two sections of the noncommutative Chern-Simons theory, its matrix decription, and the finite dimensional matrix version proposed by Polychronakos.

\section{Noncommutative Chern-Simons theory}

Chern-Simons theory with gauge group U(1) on noncommutative two-dimensional space is described by an action

$$
S=\frac{k}{4 \pi} \int d^{3} x \epsilon^{\mu \nu \lambda}\left(A_{\mu} \star \partial_{\nu} A_{\lambda}+\frac{2}{3} A_{\mu} \star A_{\nu} \star A_{\lambda}\right)
$$

where

$$
f \star g=e^{\frac{i}{2} \theta \epsilon^{i j} \partial_{i}^{f} \partial_{j}^{g}} f g
$$

This theory is invariant under arbitrary noncommutative gauge transformations (trivial at infinity) given by

$$
A_{\mu} \rightarrow U^{-1} \star A_{\mu} \star U+i U^{-1} \star \partial_{\mu} U
$$


as long as $k$ (the level) is an integer $[9,10]$. Note that the appearance of a cubic term in the action and the quantization of the level occurs even for the case of $U(1)$, unlike in the commutative theory.

The theory may be written in an equivalent form by choosing the gauge $A_{0}=0$ in which case the action becomes

$$
S=\frac{k}{4 \pi} \int d^{3} x \epsilon^{i j} A_{i} \partial_{t} A_{j}
$$

while the equation of motion for $A_{0}$ must be imposed as a constraint,

$$
F_{i j}=\partial_{i} A_{j}-\partial_{j} A_{i}-i A_{i} \star A_{j}+i A_{j} \star A_{i}=0
$$

It turns out that this action and constraint also arise from a matrix model in $0+1$ dimensions, given by

$$
S=\frac{k}{\theta} \int d t \operatorname{Tr}\left(\frac{1}{2} \epsilon^{i j} D X^{i} X^{j}\right)+k \int d t \operatorname{Tr}(A)
$$

where $\mathrm{X}$ and $\mathrm{A}$ are hermitian matrices $[12,13,14]$. The action is invariant under gauge transformations

$$
X^{i} \rightarrow U^{-1} X^{i} U, \quad A \rightarrow U^{-1} A U+i U^{-1} \partial_{t} U
$$

as long as $U$ is taken to be trivial at $t= \pm \infty$ and $k$ is an integer. We may choose the gauge $A=0$ in which case the action becomes

$$
S=\frac{k}{\theta} \int d t \operatorname{Tr}\left(\frac{1}{2} \epsilon^{i j} \dot{X}^{i} X^{j}\right)
$$

while the equation of motion for $\mathrm{A}$ is

$$
\left[X^{1}, X^{2}\right]=i \theta \mathbb{1}
$$

which must be taken as a constraint (note that the commutator here is a matrix commutator). This has no solutions for finite dimensional matrices (as may be seen by taking the trace), thus, we must take $X^{i}$ to be infinite dimensional. A particular solution is $X^{i}=y^{i}$, where $y^{1}$ and $y^{2} / \theta$ are the usual matrices representing $x$ and $p$ in the harmonic oscillator basis. Expanding the action (16) and constraint (17) about this classical solution, $X^{i}=y^{i}+\theta \epsilon^{i j} A_{j}$ and considering $A_{j}$ to be functions of the noncommuting coordinates $y^{i}$ gives precisely the Lagrangian (13) with the constraint (14). ${ }^{1}$ Thus, the matrix model

\footnotetext{
${ }^{1}$ This step requires making the transition from the operator formalism for fields on noncommutative space to the representation in terms of ordinary functions multiplied by the star products. Explicitly, we have

$$
\left[y_{i}, f\right] \rightarrow i \theta \epsilon_{i k} \partial_{k} f, \quad \operatorname{Tr}\left(f_{1} \cdots f_{n}\right) \rightarrow \frac{1}{2 \pi \theta} \int d^{2} x\left(f_{1} \star \cdots \star f_{n}\right)
$$
}

For more details of the relationship between these two representations, see for example [11]. 
(15), expanded about the background $X^{i}=y^{i}$ is equivalent to the noncommutative $U(1)$ Chern-Simons theory. To be precise, we should restrict to fluctuations $A_{i}$ about the background described by compact operators (this is equivalent to the condition that the fields vanish at infinity in the field theory language) with a similar condition on the allowed gauge transformations [11]. Interestingly, the same matrix model expanded about a different background $X^{i}=y^{i} \otimes \mathbb{1}_{N \times N}$ gives the $U(N)$ noncommutative Chern-Simons theory (this highlights the need to restrict the allowed $X$ 's to a specific subset of all hermitian matrices before the matrix model gives a well defined theory).

Starting from the matrix action (16), canonical quantization gives the commutation relations

$$
\left[X_{m n}^{1}, X_{p q}^{2}\right]=i \frac{\theta}{k} \delta_{m q} \delta_{n p} .
$$

Thus, we may choose the wavefunction to be a function of the matrix $X=X^{1}$ and represent $X^{2}$ as

$$
X_{m n}^{2}=\frac{\theta}{k} i \frac{\partial}{\partial X_{n m}} .
$$

The constraint (17) should then be imposed as an operator constraint on the wavefunction. With the proper operator ordering, the left side of (17) generates $U(N)$ transformations on the wavefunction [4], and it follows that the constraint is equivalent to the condition

$$
\Psi\left(U^{-1} X U\right)=(\operatorname{det} U)^{k} \Psi(X) .
$$

Since the Hamiltonian for the theory is zero, this equation provides all the information about the allowed wavefunctions. ${ }^{2}$

Because the matrices are infinite dimensional, it is difficult to determine solutions to this constraint or even to understand whether it has well defined solutions without some sort of regularization. However, as pointed out in [4] this constraint already provides some direct evidence for a connection with the Laughlin states. Specifically, if $U$ is taken to be a permutation matrix which would permute two elements of a diagonal matrix, then we have $\operatorname{det}(U)=-1$, so (18) indicates that the wavefunction obeys fermionic statistics for odd values of $k$ and bosonic statistics for even values of $k$. Identifying $k$ with the inverse filling fraction in the Laughlin theory, this gives precisely the relation between statistics and filling fraction obeyed in the Laughlin wavefunctions.

In order to proceed further, it is very helpful to work with a regulated version of the theory that is consistent with finite dimensional matrices. Such a theory has been proposed by Polychronakos [5]. In the next section, we review his construction, and in section 5 determine the complete set of allowed wavefunctions for the theory.

\footnotetext{
${ }^{2}$ However, as discussed above, we must be careful to restrict to an appropriate subset of allowed $X$ 's and $U$ 's before the theory is well defined.
} 


\section{Finite N Matrix Chern-Simons theory}

In [5], Polychronakos proposed a modification of the action (16) to make it consistent with matrices of arbitrary finite dimension $N$. He suggested that the resulting action should provide a description of the states of a quantum hall droplet of finite extent composed of $N$ electrons. Further, he showed that many of the expected excitations of a quantum hall droplet, including area-preserving boundary excitations and quasiholes with fractional charge $1 / k$, appear naturally in the model.

The action proposed by Polychronakos is

$$
S=\frac{k}{2 \theta} \int d t \operatorname{Tr}\left(\epsilon^{i j} D X^{i} X^{j}-\omega X_{i}^{2}\right)+i \int d t \Psi^{\dagger} D \Psi+k \int d t \operatorname{Tr}(A) .
$$

Relative to the orginal matrix model (16), there are two additional terms. The first is a potential term $-\omega X_{i}^{2}$ analogous to the harmonic oscillator potential considered in section 2. Again, this serves to break the degeneracy arising from translation invariance and also provides a Hamiltonian for the theory that selects a unique ground state.

The other new term involves complex bosons $\Psi_{i}$ transforming in the fundamental representation of the gauge group. With the additional $\Psi_{i}$ term, the constraint becomes

$$
\left[X^{1}, X^{2}\right]_{m n}+\frac{i \theta}{k} \Psi_{m} \Psi_{n}^{\dagger}=i \theta \delta_{m n}
$$

This is now consistent with finite dimensional $X^{i}$ since the left side is no longer traceless. For $N \times N$ matrices the trace of this equation gives

$$
\Psi_{m}^{\dagger} \Psi_{m}=N k
$$

As demonstrated in [5] the constraints may be explicitly solved classically by using the $U(N)$ symmetry to make $X^{1}$ diagonal and $\Psi$ real. The resulting system, with no remaining gauge symmetry apart from the permutations, contains $N$ real degrees of freedom, the same number as $N$ electrons in the lowest Landau level. ${ }^{3}$

As discussed in [5], a useful way to understand the relationship of this theory to the original Chern-Simons theory is to note that in a particular gauge, we may solve the trace part of constraint (20) by taking

$$
\Psi_{i}=(0,0, \cdots, \sqrt{k N}) .
$$

The resulting theory, with the harmonic oscillator potential taken to zero, retains a $U(N-$ $1) \times U(1)$ symmetry, and is described by the Lagrangian (16) and the constraint (17) modified so that the $(N, N)$ entry of the right hand side becomes $i \theta(1-N)$. This is

\footnotetext{
${ }^{3}$ In fact, as discussed in [5] the theory obtained in this way is precisely the Calogero model for $N$ particles in one dimension.
} 
essentially the minimal modification of the original matrix Chern-Simons theory to make it consistent with finite dimensional matrices. In the $N=\infty$ limit, the constraint equation formally goes over to the original one (17) so it seems reasonable to claim that the $N=$ $\infty, \omega \rightarrow 0$ limit of the Polychronakos model is noncommutative Chern-Simons theory. Alternately, we may think of this limit as a particular way of giving a well defined definition of non-commutative Chern-Simons theory.

Let us now consider the finite matrix Chern-Simons theory quantum mechanically, following [5]. Rather than solving the constraint before quantization, we will proceed by first quantizing the theory and then applying the constraints as operator conditions on the wavefunction. It is convenient to introduce the matrix $A \equiv \sqrt{\frac{k}{2 \theta}}(X+i Y)$. Then the canonical commutation relations give

$$
\left[A_{i j}, A_{k l}^{\dagger}\right]=\delta_{i l} \delta_{j k} \quad\left[\Psi_{i}, \Psi_{j}^{\dagger}\right]=\delta_{i j} .
$$

Thus, we have $N^{2}$ creation and annihilation operators coming from the $A$ 's and $N$ creation and annihilation operators coming from the $\Psi$ 's. The hamiltonian is given by

$$
H=\omega\left(\frac{N^{2}}{2}+\mathcal{N}_{A}\right)
$$

where $\mathcal{N}_{A}=\sum A_{i j}^{\dagger} A_{j i}$ is the number operator associated with the $A$ 's. Thus, energy eigenstates will be linear combinations of terms with a fixed number of $A^{\dagger}$ creation operators acting on the Fock space vacuum. Examining the trace part of the constraint (20), we see that the left hand side is simply the number operator $\mathcal{N}_{\Psi}$ associated with the $\Psi$ creation and annihilation operators, so all wavefunctions must have a fixed number $N k$ of $\Psi^{\dagger}$ creation operators acting on the Fock space vacuum. Finally, as shown in [5], the traceless part of the constraint (19) demands that the wavefunction be invariant under $S U(N)$ transformations, under which the creation operators transform in the adjoint and antifundamental,

$$
A^{\dagger} \rightarrow U A^{\dagger} U^{\dagger}, \quad \Psi^{\dagger} \rightarrow \Psi^{\dagger} U^{\dagger}
$$

Therefore, as pointed out in [5] the problem reduces to a group theory problem of determining all ways of combining $N k$ antifundamentals (symmetrized) and any fixed number of adjoints to form a singlet of $S U(N)$. In the next section we solve this problem explicitly and write down the complete set of wavefunctions for the model. We will see that they bear a striking resemblance to the Laughlin-type wavefunctions considered in section 2 and then argue that the two systems are in fact equivalent.

\section{Determining the wavefunctions}

From the results of the previous section, any wavefunction describing an energy eigenstate will be a sum of terms of the form

$$
A_{j_{1}}^{\dagger i_{1}} \cdots A_{j_{M}}^{\dagger i_{M}} \Psi_{l_{1}}^{\dagger} \cdots \Psi_{l_{N k}}^{\dagger}|0\rangle
$$


with $N k$ creation operators $\Psi^{\dagger}$ and a fixed number $M$ of $A^{\dagger}$ creation operators acting on the Fock space vacuum, such that the set of terms forms a singlet under the $S U(N)$ transformations (22). Here, we have written fundamental indices as upper indices and antifundamental indices as lower indices. The problem of forming a singlet out of the $N k$ antifundamentals and $M$ adjoints is basically just a matter of contracting all the indices using the invariant tensors of $S U(N)$. In particular, we may contract any upper index with any lower index using $\delta_{j}^{i}$, or we may contract any set of $\mathrm{N}$ lower indices (or $\mathrm{N}$ upper indices) with an $N$-index completely antisymmetric $\epsilon$ tensor. We may place the further restriction that only one type of epsilon tensor may appear (either upper or lower index) since the product of an upper index epsilon tensor and a lower index epsilon tensor may be rewritten as a sum of products of $2 N$ delta functions.

Let us consider first the indices on the $N k \Psi^{\dagger} \mathrm{s}$. The lower index on each $\Psi^{\dagger}$ must contract either with the upper index on an $A^{\dagger}$ or with an epsilon tensor. If the $\Psi^{\dagger}$ contracts with an $A^{\dagger}$, the resulting object will again have a single lower index. Repeating this logic, we conclude that each $\Psi^{\dagger}$ will contract with some number of $A^{\dagger}$ s and that the resulting object will have its single lower index contracted with an (upper index) epsilon tensor.

On the other hand, consider a given upper index on an epsilon tensor. This will be contracted either with a $\Psi^{\dagger}$ or with the lower index on an $A^{\dagger}$, leaving another upper index. In the second case, the new upper index will either contract with a $\Psi^{\dagger}$ or another $A^{\dagger}$, etc..., so it is clear that a given upper index on an epsilon tensor will contract with some number of $A^{\dagger}$ s and then with a $\Psi^{\dagger}$.

The previous two paragraphs show that the lower indices on the $N k \Psi^{\dagger}$ s and the upper indices on the epsilon tensors (there must be $k$ of them) must pair up completely (with arbitrary numbers of intermediate $A^{\dagger} \mathrm{s}$ ) giving $k$ blocks of the form

$$
\epsilon^{i_{1} \cdots i_{N}}\left(\Psi^{\dagger} A^{\dagger n_{1}}\right)_{i_{1}} \cdots\left(\Psi^{\dagger} A^{\dagger n_{N}}\right)_{i_{N}}
$$

Because of the antisymmetry of the $\epsilon$ tensor, this expression will vanish unless all of the $n_{i}$ 's are different, so without loss of generality, we may take $0 \leq n_{1}<\cdots<n_{N}$ in each of the blocks.

We have now accounted for all $\Psi^{\dagger}$ s and epsilon tensors, but we may have some additional $A^{\dagger} \mathrm{s}$ with indices contracted amongst themselves. Such terms will be generated by products of $\operatorname{Tr}\left(A^{\dagger}\right), \ldots, \operatorname{Tr}\left(A^{\dagger N}\right) .{ }^{4}$ Thus, a given singlet wavefunction may also include a set of $A^{\dagger} \mathrm{s}$ of the form

$$
\left(\operatorname{tr} A^{\dagger N}\right)^{c_{N}} \cdots\left(\operatorname{tr} A^{\dagger}\right)^{c_{1}} .
$$

To summarize, a general $S U(N)$ singlet energy eigenstate wavefunction will be built out of terms with identical numbers of $A^{\dagger}$ s each containing $k$ blocks of the form (23) plus a block of the form (24) acting on the Fock space vacuum. Actually, this description is rather redundant, and using Fact 3 from the appendix, it is straightforward to obtain the following two minimal bases of these singlet energy eigenstate wavefunctions.

\footnotetext{
${ }^{4}$ Note that $\operatorname{det}\left(A^{\dagger}\right)$ as well as traces of higher powers of $A^{\dagger}$ may be written in terms of these.
} 
The first basis is obtained by restricting to $c_{i}=0$ in (24), taking $n_{i}=i-1$ in $k-1$ of the blocks (23) and taking $n_{i}$ arbitrary (but distinct) in the $k$ th block to get

$$
\left|\left\{n_{i}\right\}, k\right\rangle=\epsilon^{i_{1} \cdots i_{N}}\left(\Psi^{\dagger} A^{\dagger n_{1}}\right)_{i_{1}} \cdots\left(\Psi^{\dagger} A^{\dagger n_{N}}\right)_{i_{N}}\left(\epsilon^{i_{1} \cdots i_{N}}\left(\Psi^{\dagger} A^{\dagger 0}\right)_{i_{1}} \cdots\left(\Psi^{\dagger} A^{\dagger N-1}\right)_{i_{N}}\right)^{k-1}|0\rangle ;
$$

where $0 \leq n_{1}<\cdots<n_{N}$. From (21), we find that these states have energy levels $E\left(\left\{n_{i}\right\}, k\right)=\omega\left(\frac{N}{2}(N+(k-1)(N-1))+\sum n_{i}\right)$.

An alternative basis is obtained by fixing $n_{i}$ at their minimum values $n_{i}=i-1$ in each block (23) and taking $c_{i}$ arbitrary in (24),

$$
\left|\left\{c_{i}\right\}, k\right\rangle=\left(\operatorname{tr} A^{\dagger N}\right)^{c_{N}} \cdots\left(\operatorname{tr} A^{\dagger}\right)^{c_{1}}\left(\epsilon^{i_{1} \cdots i_{N}}\left(\Psi^{\dagger} A^{\dagger 0}\right)_{i_{1}} \cdots\left(\Psi^{\dagger} A^{\dagger N-1}\right)_{i_{N}}\right)^{k}|0\rangle
$$

The energy levels for these states are given by $E\left(\left\{c_{m}\right\}, k\right)=\omega\left(\frac{N}{2}(N+k(N-1))+\sum m c_{m}\right)$. It is not difficult to verify that these energy levels and degeneracies precisely match those of $(25)$.

Each of these bases realize precisely the known energy levels and degeneracies of the model (given in [5] and references therein), so we may conclude that each gives a minimal basis of linearly independent energy eigenstate wavefunctions.

We now compare the states (25) and (26) to the Laughlin-type wavefunctions (11) and (12) respectively that we constructed in section 2 . We see that the states have been labeled in an identical fashion, and that states with the same label have the same energy level relative to the respective ground states (with the identification $\omega=\frac{\kappa}{B}$ ). More strikingly, the similarly labeled states have almost precisely the same form. In particular, the formal substitution $\Psi_{i}^{\dagger} \rightarrow 1, A_{i j}^{\dagger} \rightarrow \delta_{i j} a_{i}^{\dagger}$ maps a given state in (25) or (26) precisely to the similarly labeled state in (11) or (12).

This comparison makes it obvious that the level $k$ finite matrix Chern-Simons theory has exactly the same energy levels and the same number of states at each energy level as the theory of $N$ composite fermions in the lowest Landau level. This guarantees that the two theories are equivalent, since for each energy level, we can find an orthogonal basis of wavefunctions in each of the theories and then define an isomorphism that maps the orthogonal bases into each other. In this way, we generate an isomorphism between the Hilbert spaces in the two theories that preserves the inner product structure and maps the respective Hamiltonians into each other. In other words, the finite $N$, level $k=2 p+1$ matrix Chern-Simons theory is precisely equivalent to the theory of $N$ (noninteracting) composite fermions with $2 p$ attached flux lines in the Lowest Landau level, which is believed to accurately describe the filling fraction $1 / k$ state of $N$ electrons and its excitations. ${ }^{5}$

It should be noted that despite the formal similarity between the bases (25), (26) and (11), (12), the similarly labeled states do not precisely map into one another under the

\footnotetext{
${ }^{5}$ The equivalence has been established with the inclusion of a harmonic oscillator potential in each model to break the translation invariance, but the strength of this may be taken to zero if desired.
} 
isomorphism. For example, at $k=1$ the basis (11) is orthogonal, while we have checked that two states of the basis (25) in the second excited energy level are non-orthogonal. However, given the explicit wavefunctions we have derived, it is straightforward to determine explicitly how the isomorphism acts on the bases we have derived at a given energy level.

In any case, the ground state wavefunctions map into one another, so the Laughlin wavefunction for filling fraction $1 / k$ is equivalent to the wavefunction

$$
\left|0_{k}\right\rangle=\left(\epsilon^{i_{1} \cdots i_{N}}\left(\Psi^{\dagger} A^{\dagger 0}\right)_{i_{1}} \cdots\left(\Psi^{\dagger} A^{\dagger N-1}\right)_{i_{N}}\right)^{k}|0\rangle
$$

of the matrix Chern-Simons theory.

It is interesting to note that the equivalence we have described is related to the work of $[15,16]$ on the Calogero model [17]. They showed that the Calogero model for a certain range of parameters could be identified with the physics of anyons (particles with fractional statistics) in the lowest Landau level (originally conjectured in [18]). On the other hand, it has been shown $[19,20]$ that the finite matrix models we have considered are equivalent to the Calogero model with certain discrete values of the parameters (though not values appropriate for describing anyons). Thus, the equivalence we have demonstrated is related to two previously known equivalences, though our system does not contain particles with fractional statistics, but rather composite fermions with a sort of "higher order" integer statistics (higher order zeros in the wavefunction when particles become coincident).

\section{Discussion}

We have established a precise equivalence between the finite $N$ matrix Chern-Simons theory and the theory of $N$ composite fermions in the lowest Landau level, believed to accurately describe the filling fraction $1 / k$ fractional Quantum hall state of $N$ electrons. To be precise, the latter theory is defined to be the theory of $N$ non-interacting fermions in the lowest Landau level with the constraint that the wavefunctions have a zero of order $\geq k$ whenever two of the particles become coincident.

In the large $N$ limit, the composite fermion theory should provide a good description of the $\nu=1 / k$ quantum Hall fluid of infinite extent, since the boundary of the quantum Hall droplet goes off to infinity. On the other hand, as discussed in section 4, we expect that the large $N$ matrix Chern Simons theory (with the harmonic oscillator potential turned off) should be equivalent to the original noncommutative Chern-Simons field theory. Thus, as conjectured by Susskind, the noncommutative field theory at level $k$ provides a good description of the of the filling fraction $1 / k$ state of electrons in a strong magnetic field, and in particular is precisely equivalent to the Laughlin theory (and its present understanding as describing noninteracting composite fermions in the lowest Landau level). 


\section{Acknowledgements}

We would especially like to thank Lenny Susskind for many valuable discussions out of which this project originated. We have also benefitted from discussions with Michal Fabinger, Matt Kleban, John McGreevy, and Nick Toumbas. M.V.R. would like to thank the Institute for Theoretical Physics in Santa Barbara for hospitality during the final stages of this work. The work of M.V.R. is supported in part by the Stanford Institute for Theoretical Physics and by NSF grant 9870115. The work of Simeon Hellerman is supported by the D.O.E. under contract DE-AC03-76SF00098 and by a D.O.E. OJI grant.

\section{A Facts to obtain a minimal basis of wavefunctions.}

In section 5, we derived an exhaustive set of wavefunctions describing energy eigenstates of the finite matrix Chern-Simons theory. In this appendix, we derive a number of facts that help to determine a minimal basis of these wavefunctions.

Fact 1: Defining

$$
A(z) \equiv \prod_{i<j}\left(z_{i}-z_{j}\right)
$$

we have

$$
A(z)=\epsilon^{i_{1} \cdots i_{N}} z_{i_{1}}^{0} \cdots z_{i_{N}}^{N-1} .
$$

Proof: From the definition, it is clear that $A$ is the lowest order polynomial that is completely antisymmetric in its arguments, since any such polynomial must have a factor $\left(z_{i}-z_{j}\right)$ for each $i \neq j$. The second expression is also completely antisymmetric and has the same order, so it must equal $A$ up to a numerical factor which is easily checked to be 1 .

Fact 2: Any polynomial

$$
f(z)=\epsilon^{i_{1} \cdots i_{N}} z_{i_{1}}^{n_{1}} \cdots z_{i_{N}}^{n_{N}}
$$

may be written as a sum of terms of the form

$$
g(z)=S_{N}^{c_{N}} \cdots S_{1}^{c_{1}} A(z)
$$

where $S_{k}=\sum_{i} z_{i}^{k}$. Conversely, any polynomial of the form (28) may be written as a sum of terms of the form (27).

Proof: The polynomial $f(z)$ is completely antisymmetric in its arguments, thus it vanishes when any two of its arguments are set equal. This implies that $A$ divides $f$, so $f=S A$ for some polynomial $S$. Since $f$ and $A$ are both totally antisymmetric, $S$ must be totally 
symmetric. It is not difficult to show that any symmetric polynomial $S\left(z_{1}, \ldots, z_{N}\right)$ may be written uniquely as a sum of terms of the form

$$
S_{N}^{c_{N}} \cdots S_{1}^{c_{1}}
$$

where $S_{k}=\sum_{i} z_{i}^{k}$. To prove the converse, we simply note that polynomials of the form (28) are completely antisymmetric and are thus allowed coherent state wavefunctions for $\mathrm{n}$ fermions in a harmonic oscillator potential. On the other hand, as pointed out in section 2, polynomials of the form (27) represent the coherent state wavefunctions for an orthogonal basis of such states.

Fact 3: Let $\Psi^{\dagger}$ and $A^{\dagger}$ be an $N$-dimensional vector and an $N \times N$ matrix of commuting variables. Then any expression of the form

$$
F\left(\Psi^{\dagger}, A^{\dagger}\right)=\epsilon^{i_{1} \cdots i_{N}}\left(\Psi^{\dagger} A^{\dagger n_{1}}\right)_{i_{1}} \cdots\left(\Psi^{\dagger} A^{\dagger n_{N}}\right)_{i_{N}}
$$

may be written uniquely as a sum of terms of the form

$$
G\left(\Psi^{\dagger}, A^{\dagger}\right)=\left(\operatorname{tr} A^{\dagger N}\right)^{c_{N}} \cdots\left(\operatorname{tr} A^{\dagger}\right)^{c_{1}} \epsilon^{i_{1} \cdots i_{N}}\left(\Psi^{\dagger} A^{\dagger 0}\right)_{i_{1}} \cdots\left(\Psi^{\dagger} A^{\dagger N-1}\right)_{i_{N}}
$$

Conversely, any expression of the form (30) may be written uniquely as a sum of terms of the form (29).

Proof: First note that there is a one-to-one correspondence between expressions of the form (29) and polynomials of the form (27) obtained by taking the same $n_{i}$, and a one-toone correspondence between expressions of the form (30) and polynomials of the form (28) obtained by taking the same $c_{i}$. By Fact 2 , given any $F$, the corresponding polynomial $f$ may be written uniquely as a sum of terms of the form (28) which we denote $\sum g_{i}$. We now prove that

$$
F\left(\Psi^{\dagger}, A^{\dagger}\right)=\sum G_{i}\left(\Psi^{\dagger}, A^{\dagger}\right)
$$

where $G_{i}$ is the expression corresponding to $g_{i}$. First, note that for $A_{i j}^{\dagger}=\delta_{i j} z_{i}$ we have

$$
F\left(\Psi^{\dagger}, A^{\dagger}\right)=f\left(z_{i}\right) \prod \Psi_{j}^{\dagger}=\sum g_{i}\left(z_{i}\right) \prod \Psi_{j}^{\dagger}=\sum G_{i}\left(\Psi^{\dagger}, A^{\dagger}\right) .
$$

Next, let $A^{\dagger}$ be an arbitrary hermitian matrix of complex numbers. Then $A^{\dagger}=U D U^{\dagger}$ for some unitary matrix $U$ and real diagonal matrix $D$. It is straightforward to check using $(32)$,

$$
F\left(\Psi^{\dagger}, A^{\dagger}\right)=F\left(\Psi^{\dagger} U, D\right)=\sum G_{i}\left(\Psi^{\dagger} U, D\right)=\sum G_{i}\left(\Psi^{\dagger}, A^{\dagger}\right) .
$$

Now, we have shown that (31) holds for arbitrary $\Psi^{\dagger}$ and any hermitian matrix $A^{\dagger}$. But both sides of (31) are simply finite polynomials in the components of $\Psi^{\dagger}$ and $A^{\dagger}$ (and not their Hermitian or complex conjugates). Therefore the equivalence clearly cannot depend on $A^{\dagger}$ being Hermitian so it must hold for any numerical matrix $A^{\dagger}$ and vector 
$\Psi^{\dagger}$. This implies that the two polynomials are equivalent for all numerical values of their arguments, so we may conclude that (31) is true as an identity between polynomials of commuting variables. The fact that the decomposition (31) is unique follows immediately from the uniqueness in fact 2 . Thus, any $F$ can be written uniquely as a sum of $G$ s, and by an identical argument, we can show that any $G$ can be written uniquely as a sum of F's.

\section{References}

[1] A. Connes, M. R. Douglas and A. Schwarz, "Noncommutative geometry and matrix theory: Compactification on tori," JHEP 9802, 003 (1998) [hep-th/9711162].

[2] N. Seiberg and E. Witten, "String theory and noncommutative geometry," JHEP 9909, 032 (1999) [hep-th/9908142].

[3] S. Girvin, "The Quantum Hall Effect: Novel Excitations and Broken Symmetries," cond-mat/9907002.

[4] L. Susskind, "The quantum Hall fluid and non-commutative Chern Simons theory," hep-th/0101029.

[5] A. P. Polychronakos, "Quantum Hall states as matrix Chern-Simons theory," hepth/0103013.

[6] R. B. Laughlin, Phys. Rev. Lett. 501395 (1983).

[7] J. K. Jain, Phys. Rev. Lett. 63, 199 (1989).

[8] J. K. Jain, in "Perspectives in Quantum Hall Effects," Edited by Sankar Das Sarma and Aron Pinczuk (Wiley, New York, 1988)

[9] V. P. Nair and A. P. Polychronakos, "On level quantization for the noncommutative Chern-Simons theory," hep-th/0102181.

[10] D. Bak, K. Lee and J. Park, "Chern-Simons theories on noncommutative plane," hep-th/0102188.

[11] J. A. Harvey, "Komaba lectures on noncommutative solitons and D-branes," hepth/0102076.

[12] B. A. Bernevig, J. H. Brodie, L. Susskind and N. Toumbas, "How Bob Laughlin tamed the giant graviton from Taub-NUT space," JHEP0102, 003 (2001) [hep-th/0010105].

[13] A. P. Polychronakos, "Noncommutative Chern-Simons terms and the noncommutative vacuum," JHEP0011, 008 (2000) [hep-th/0010264]. 
[14] J. Kluson, "Matrix model and noncommutative Chern-Simons theory," hepth/0012184.

[15] L. Brink, T. H. Hansson and M. A. Vasiliev, "Explicit solution to the N body Calogero problem," Phys. Lett. B 286, 109 (1992) [hep-th/9206049].

[16] L. Brink, T. H. Hansson, S. Konstein and M. A. Vasiliev, "The Calogero model: Anyonic representation, fermionic extension and supersymmetry," Nucl. Phys. B 401, 591 (1993) [hep-th/9302023].

[17] F. Calogero, "Solution Of The One-Dimensional N Body Problems With Quadratic And/Or Inversely Quadratic Pair Potentials," J. Math. Phys. 12, 419 (1971).

[18] T. H. Hansson, J. M. Leinaas, J. Myrheim, "Dimensional Reduction in Anyon Systems," Nucl. Phys. B 384, 559 (1992)

[19] M. A. Olshanetsky and A. M. Perelomov, "Classical Integrable Finite Dimensional Systems Related To Lie Algebras," Phys. Rept. 71, 313 (1981), Phys. Rept. 94, 6 (19yy).

[20] A. P. Polychronakos, "Integrable systems from gauged matrix models," Phys. Lett. B 266, 29 (1991). 